\title{
Transport of active particles in an open-wedge channel
}

Lorenzo Caprini, ${ }^{1}$ Fabio Cecconi, ${ }^{2}$ and Umberto Marini Bettolo Marconi ${ }^{3}$

1) Gran Sasso Science Institute (GSSI), Via F.Crispi 7, I-67100 L'Aquila, Italy

2) Istituto dei Sistemi Complessi (CNR), Via Taurini 19, I-00185 Roma, Italy

${ }^{3)}$ Scuola di Scienze e Tecnologie, Università di Camerino, Via Madonna delle Carceri, I-62032, Camerino, Italy

The transport of independent active Brownian particles within a two-dimensional narrow channel, modeled as an open-wedge, is studied both numerically and theoretically. We show that the active force tends to localize the particles near the walls thus reducing the effect of the entropic force which, instead, is prevailing in the case of passive particles. As a consequence, the exit of active particles from the smaller side of the channel is facilitated with respect to their passive counterpart. By continuously re-injecting particles in the middle of the wedge, we obtain a steady regime whose properties are investigated with and without the presence of an external constant driving field. We characterize the statistics and properties of the exit process from the two opposite sides of the channel, also by making a comparison between the active and passive case. Our study reveals the existence of an optimal value of the persistence time of the active force which is able to guarantee the maximal efficiency in the transport process. 


\section{INTRODUCTION}

In the last years, the theoretical study of self-propelled microswimmers has become an important research area at the crossroads between biology, mathematics, and physics. These systems are ubiquitous in nature, typical examples being bacteria ${ }^{1}$, protozoa ${ }^{2}$, spermatozoa ${ }^{3}$ and living tissues ${ }^{4}$ and actin filaments ${ }^{5}$ to mention just a few. On the other hand, bioengineers and physicists are developing techniques to create artificial self-propelled objects, as in the case of the so-called Janus particles ${ }^{6 / 7}$.

The common feature of active particles is the existence of a self-propelling mechanism converting the environmental energy into motion 8 - The nature of such propulsion varies from one microswimmer to another, but in general determines a ballistic motion at short spatial and temporal scales and a diffusive motion at larger scales.

In order to describe the behavior of such systems, different theoretical models have been developed, among them we recall a) the Run\&Tumble (R\&T) model $\stackrel{12}{14}$, where the microswimmers alternatively perform at a given rate ballistic displacements and tumbles, i.e. random changes of the orientation of their velocity. b) a continuous model with a Langevinlike dynamics, the so-called Active Brownian particle (ABP) model[15] described in detail in Sec.II. c) the active Ornstein-Uhlenbeck particle (AOUP) model ${ }^{16}$.18. All these models show an intriguing phenomenology ranging from particle accumulation near the walls of a container $^{\frac{19}{25}}$ to the existence of non-Boltzmann probability distributions ${ }^{26} \sqrt[28]{2}$ also in the presence of acoustic traps 29130 , the appearance of negative mobility in the presence of non convex potentials ${ }^{31}$ and motility induced phase separation (MIPS) $)^{32}+38$, in the case of interacting active particles. The influence of geometrical constraints on the motion of active particles is a less explored and only partly understood issue, in spite of its importance in elucidating how some systems of biological interest behave. For instance, living bodies harbor colonies of bacteria normally localized in the skin, external mucosae, gastrointestinal tracts etc. These bacteria, by crossing narrow constrictions, are able to invade/infect the hosts' internal tissues that instead need to remain sterile $e^{39}$. How this passage occurs is a problem of great relevance for evident reasons, especially in cases of pathogen infections.

In the framework of R\&T modeling, first-passage properties have been studied both with 40 and without ${ }^{4142}$ thermal noise for a one-dimensional channel, whereas the same problem was numerically studied for a one-dimensional version of the ABP-mode ${ }^{43}$ and in two- 


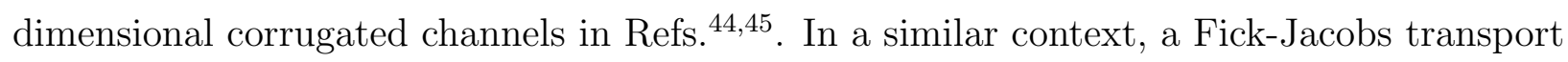
equation $\frac{46}{46}$ accounting for the channel geometry via an entropic effective force ${ }^{47 \sqrt[49]{49}}$ has been proposed for weakly active particles 50 .

In the present paper, we idealize the motion of bacteria by means of ABP and model the pore as a narrow wedge-shaped capillary. At variance with previous works, we investigate how the first-passage process of an ABP through a narrow constriction depends on the activity parameters as well as the geometry. The distribution of the escape events has been addressed by several authors $\$ 3151 \mid 53$, in this work we focus on the general features of escape process, in particular comparing the efficiency of "active" transport with the Brownian transport.

The paper is organized as follows: in Sec.II we introduce the model, while in Sec III the steady-state properties of active particles in the channel are discussed, including the density along the transport direction and the density along a section of the pore. In Sec IV, we study the escape-time statistics showing how the efficiency of the active transport depends on the active force. Finally, we summarize the main results in the conclusive section.

\section{MODEL OF ACTIVE PARTICLES IN OPEN WEDGE-GEOMETRY}

We consider an assembly of independent active particles immersed in a viscous solvent and constrained to move in the two dimensional truncated-wedge channel shown in Fig.1. We neglect the inertial effect and consider the over-damped dynamics of the particles, where the position of each particle, $\mathbf{r}$, moves according to the following stochastic differential equation

$$
\gamma \dot{\boldsymbol{r}}=\boldsymbol{F}(\mathbf{r})+\epsilon \hat{\mathbf{x}}+\gamma U_{0} \hat{\mathbf{e}}(t)
$$

where $\boldsymbol{F}$ is an external force, $\epsilon \hat{\mathbf{x}}$ a drift along the channel axis representing a systematic bias associated to a drag or a biological bias towards positive $x$. The constant $\gamma$ is the friction coefficient. The last term of Eq.(1) represents the ABP self-propulsion mechanism, namely a force of fixed strength, $\gamma U_{0}$, and varying orientation, $\hat{\mathbf{e}}(t)=(\cos \theta(t), \sin \theta(t))$, whose angle $\theta(t)$ evolves according to the following Wiener process:

$$
\dot{\theta}=\sqrt{2 D_{r}} \xi
$$

where the constant $D_{r}$ is the rotational diffusion coefficient and $\xi$ a white noise with zero average and unitary variance. As several experimental studies indicate $\sqrt[54]{ }$, the influence of 


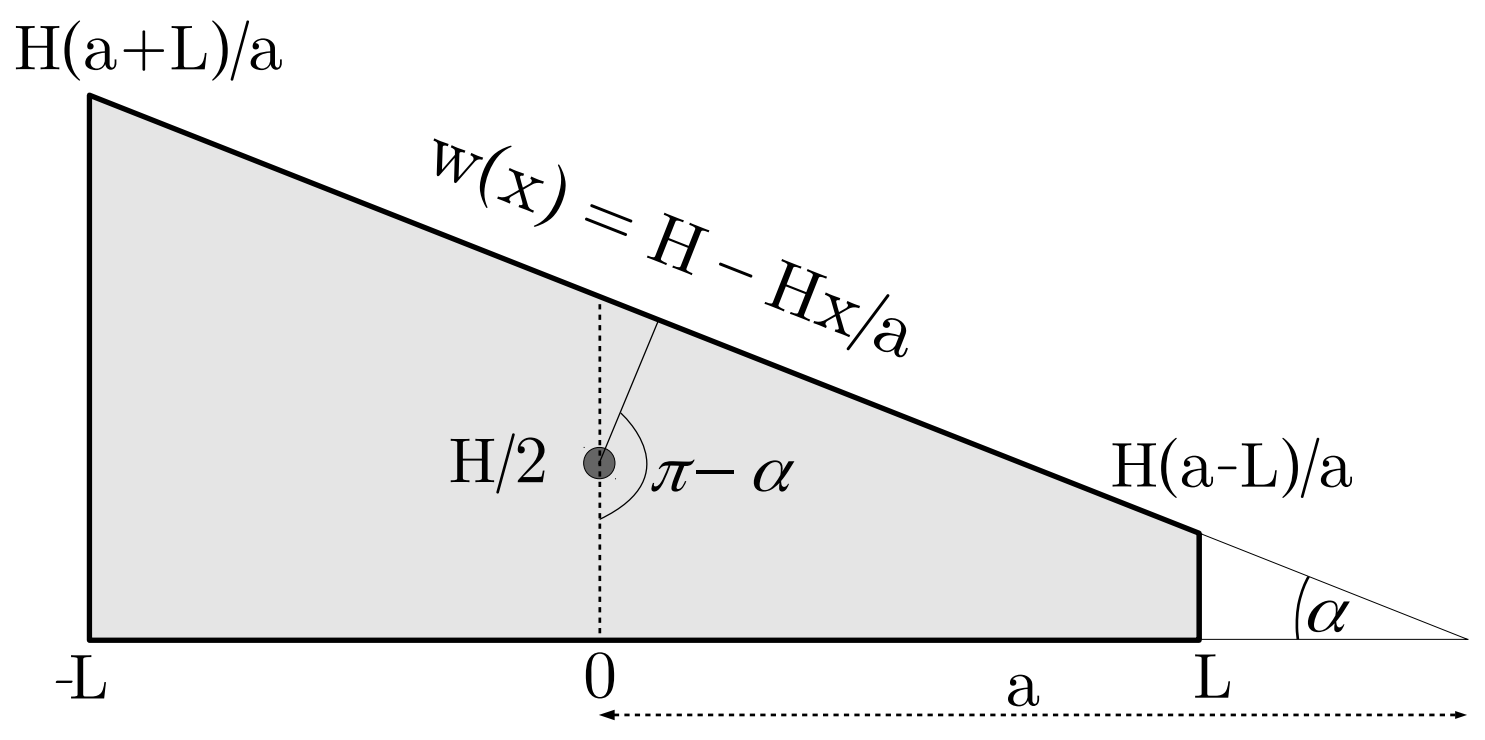

FIG. 1. Sketch of the truncated wedge used as simulation box $Q=\{|x| \leq L, 0 \leq y \leq w(x)\}$, bounded by the lines $y=H-H x / a$ and $y=0$. Absorbing conditions are placed at $x= \pm L$, for which the particles crossing $x= \pm L$ are removed from the system. The black circle, centered in $(0, H / 2)$, marks the narrow region where particles are either initially emitted or re-injected after their absorption.

the thermal agitation of the solvent surrounding the microswimmers can be neglected 5 .

The particles are confined to the domain $Q=\{(x, y):|x| \leq L, 0 \leq y<w(x)\}$ bounded by the bottom of the open-wedge channel at $y=0$ and by its upper boundary

$$
w(x)=\frac{H}{a}(a-x) .
$$

The left and right vertical boundaries, at $x= \pm L$, are absorbing, while both boundaries are soft reflecting walls (no-flux boundaries). Moreover, we are interested in the narrow channel condition: $H \ll L$. The top wall exerts on the particles a force directed along its normal direction $\mathbf{n}=\left(w^{\prime}(x),-1\right) / \sqrt{1+w^{\prime}(x)^{2}}$, whereas the repulsion of the bottom wall is directed along $\hat{\mathbf{y}}=(0,1)$. To represent this force we introduce a wall-potential $V(u)=V_{0} / m(\sigma / u)^{m}$, where $V_{0}$ defines its energy scale and $\sigma$ its length-scale assumed to be small with respect to $H, L$ and write:

$$
\mathbf{F}=-V^{\prime}(w(x)-y) \mathbf{n}-V^{\prime}(y) \hat{\mathbf{y}}
$$

where the prime represents the derivative with respect to the argument $u$. The form of the force (4) determines specular reflection when particles "collide" with the walls. To 

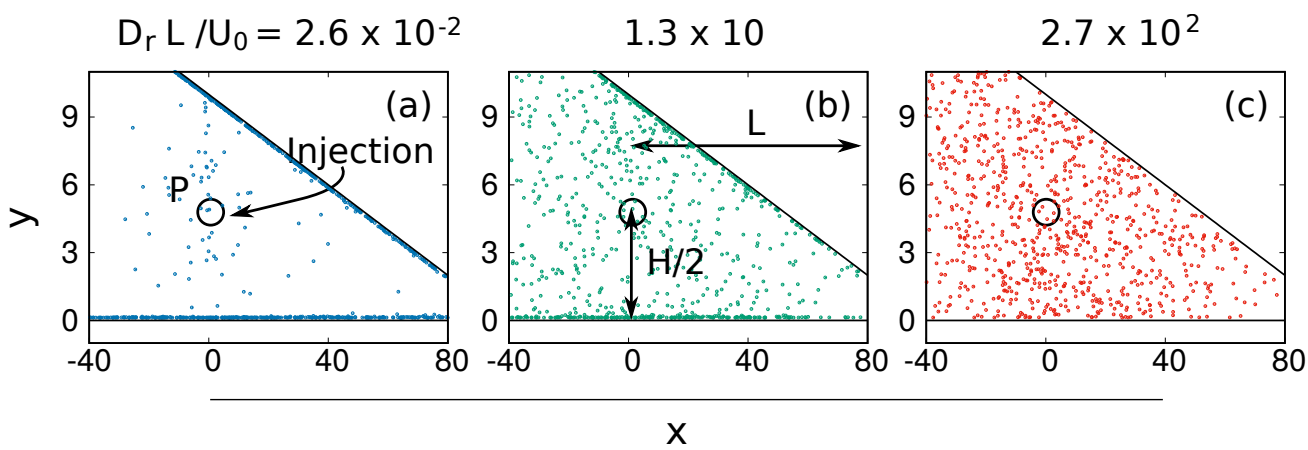

FIG. 2. Snapshots of particle configurations, upon re-injection at the site $(0, H / 2)$, for different values of the parameter $D_{r} L / U_{0}$ in the absence of field $\epsilon=0$. Panels (a), (b) and (c), showing a different degree of particle accumulation to the walls, are obtained with $D_{r} L / U_{0}=2.6 \times 10^{-2}, 1.3 \times$ $10,2.7 \times 10^{2}$, respectively. Other system parameters are $L=80, a=100, H=10$, and $U_{0}=3$.

prevent excessive penetration, the functional form of $V$ must guarantee strong repulsion when evaluated at $y=w(x)$ and $y=0$ and thus we assume $\sigma$ to be at least $\sim 10^{-2} H$ and $V_{0}=1, m=4$.

In the numerical simulations, the particles are initially placed in a small neighborhood of the point $P=(0, H / 2)$ (see Fig 1$)$, mimicking the injection by means of a "micro-pipette", and eventually leave the pore at the $L$ and $-L$ boundaries. A stationary process is achieved by reinserting these particles at the point $P$. Correspondingly, the direction of the active force acting on the re-injected particles is obtained from a uniform distribution of angles $\theta$ in the interval $[0,2 \pi]$. The evolution of $N=10^{4}$ particles is obtained by integrating Eq.(1) with a Euler-Maruyana algorithm ${ }^{\sqrt{56}}$, at least up to time $\mathcal{T} \sim 10^{3} / D_{r}$. Throughout the paper, the geometry will be fixed such that $H=10, L=80$ and $a=100$, which guarantees the condition $H \ll L$.

We begin our analysis by first discussing how the particle distribution over the domain $Q$ influences the escape properties when $D_{r}$ is varied.

\section{A. Case $\epsilon=0$}

In Fig.2 we display three snapshots of particle configuration at increasing values of $D_{r} L / U_{0}$, in the absence of an external force.

Panels a) and b) clearly show thin denser stripes near both the upper and lower wall, 

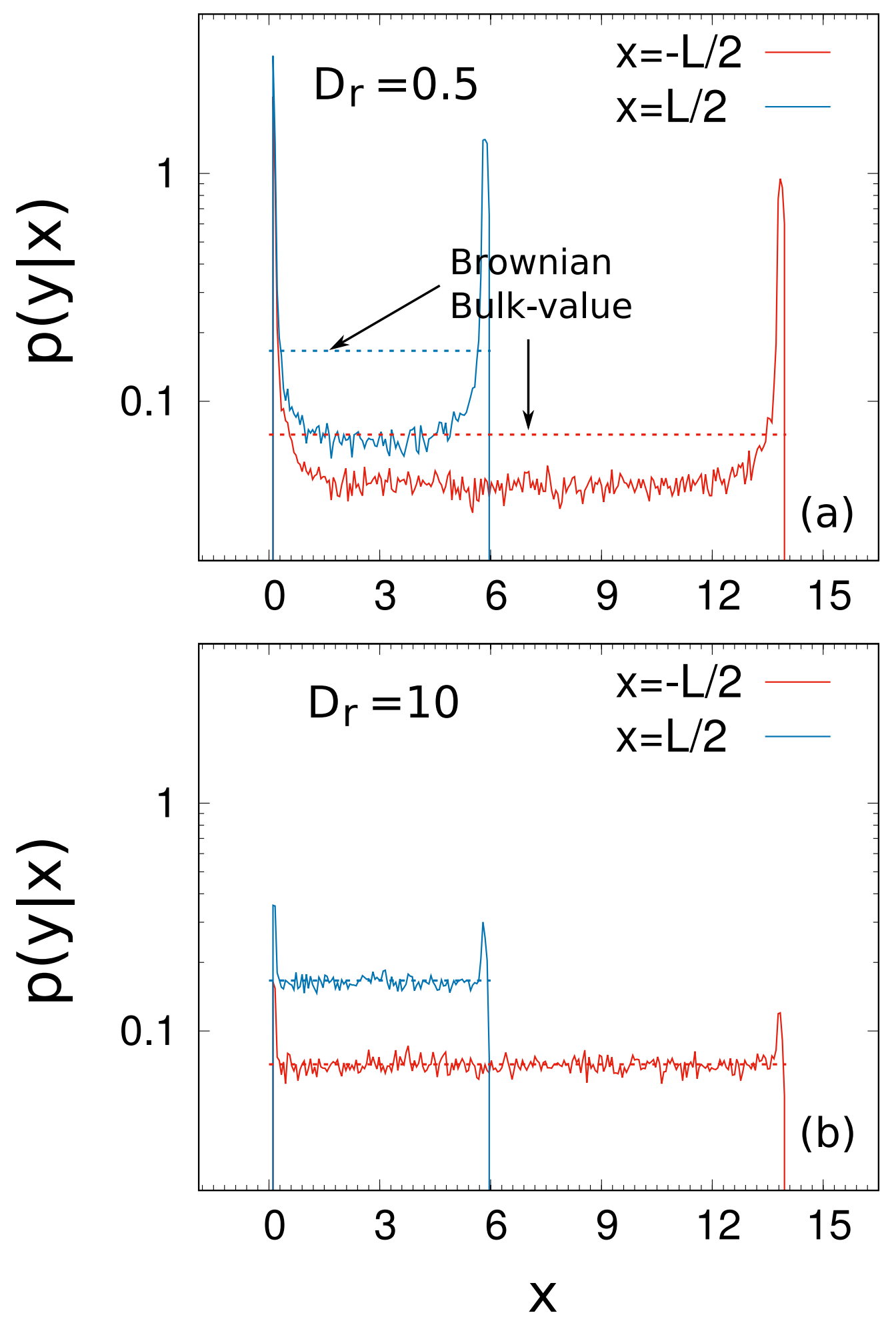

FIG. 3. Conditional probability distribution functions, $p(y \mid x)$, evaluated at $x=L / 2$ (blue line) and $x=-L / 2$ (red line). Panel a) and b) are obtained for $D_{r} L / U_{0}=1.3 \times 10,2.7 \times 10^{2}$, respectively. System parameters are: $L=80, a=100, H=10, \epsilon=0, U_{0}=3$. 


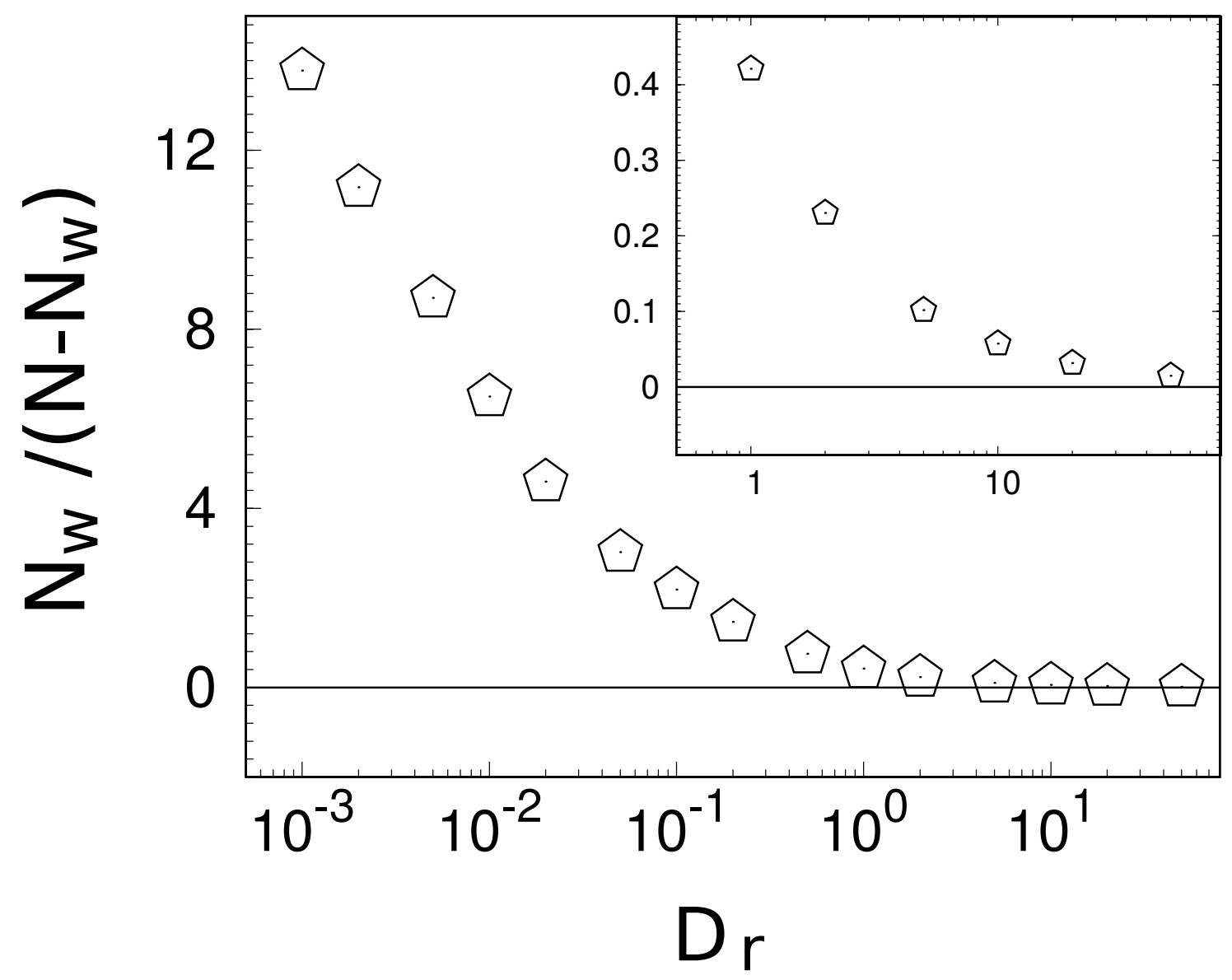

FIG. 4. Ratio $N_{w} /\left(N-N_{w}\right)$, between the number of particles accumulating at the walls and the remaining ones in the bulk, as a function of $D_{r}$. The inset is a blow-up of the range $D_{r}>1$. System parameters are the same as in Fig 3 .

indicating the tendency of strongly active particles to "climb on" confining edges. With the growth of $D_{r} L / U_{0}$, the accumulation at walls decreases from a) to b) till almost vanishing in c).

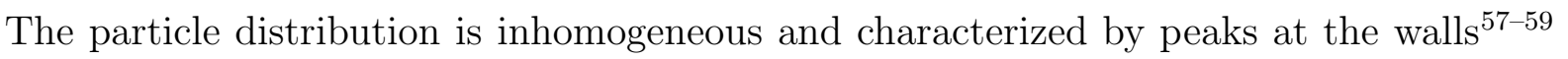
whose height is controlled by the persistence time of the force orientation, $t_{a}=1 / D_{r}^{20}$. Indeed, the larger $t_{a}$, the greater is the time spent by a particle in the proximity of the wall and the larger the accumulation ${ }^{60}$. In the limit of small persistence time $\left(D_{r} \gg \gamma\right)$, the accumulation becomes negligible and the particles behavior is quite similar to the Brownian one, with an effective temperature $T=\gamma U_{0}^{2} / 2 D_{r}^{1513216162}$.

To characterize the accumulation degree we report in Fig 3 the conditional probability distribution (pdf), $p(y \mid x)$, at two selected vertical sections centered at $x= \pm L / 2$. The 

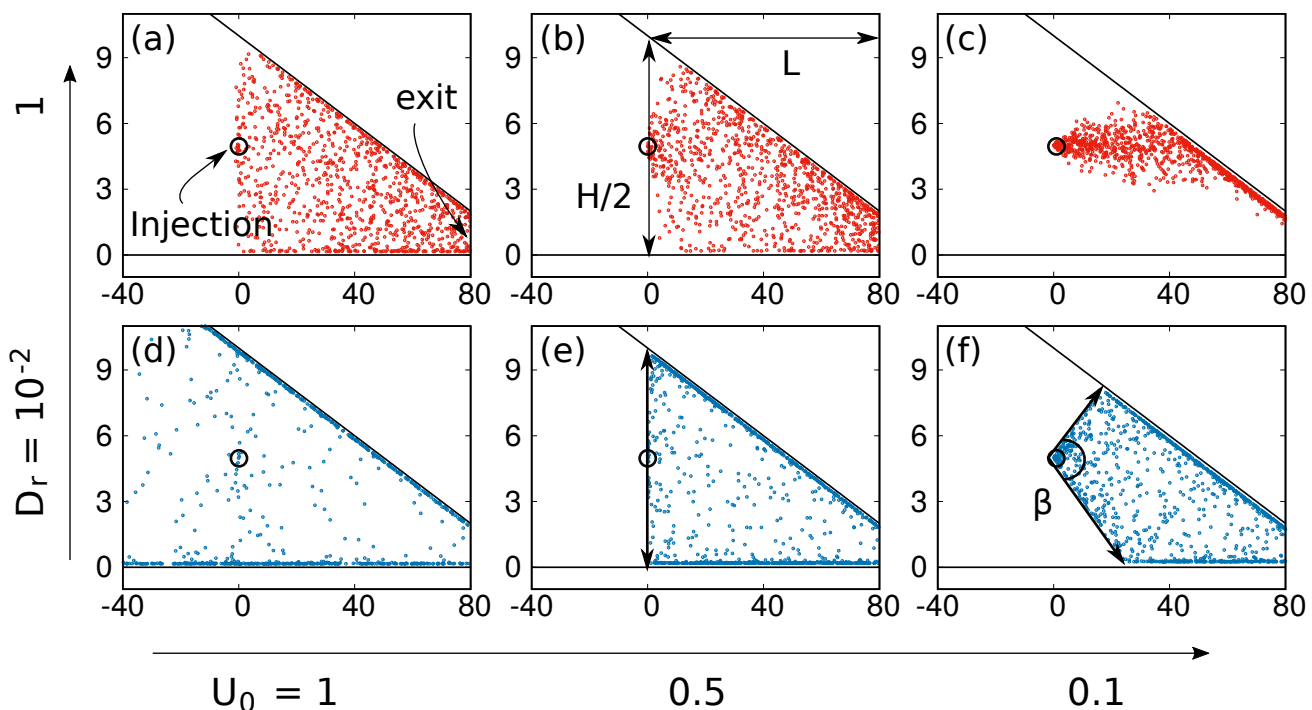

FIG. 5. Snapshots of particle positions in the presence of a field $\epsilon=0.5$ at different $U_{0}$ and $D_{r}$. Panels (a), (b) and (c) refer to values $U_{0}=1.0,0.5,0.1$ and the same $D_{r}=1.0$, while panels (d), (e) and (f) are obtained with the same set of $U_{0}$ but a value $D_{r}=0.01$. Black lines represent the walls, and the gray circle centered at $(0,5)$ is the area where particles are reinjected when they cross the right exit at $x=L=80$. The remaining parameters are $a=100$ and $H=10$.

conditional pdf of Fig. 3 a, corresponding to the snapshot b) in Fig.2, displays a bimodal behavior with well pronounced peaks due to a marked accumulation of particles at the walls. The bulk distribution, between the peaks, is not uniform, indicating that the activity not only promotes the accumulation at the boundaries, but it also influences the bulk. It is also apparent that the bulk-density is smaller than the density of Brownian system counterpart, Fig. $3 \mathrm{~b}$. This picture is in a qualitative agreement with the prediction of Malgaretti and Stark ${ }^{44}$. When $D_{r}$ becomes larger enough to determine the approach to the Brownian-like regime, see Fig 3 a referring to the snapshots c) in Fig,2, the peaks become strongly depleted, and $p(y \mid x)$ turns to be flat in the bulk, as a consequence of a fast transversal homogenization.

The accumulation also depends on the persistence length, $\lambda_{a}=U_{0} / D_{r}$, roughly the typical length-scale after which particles change direction. Indeed, the comparison between $\lambda_{a}$ and the geometrical sizes $H$ and $L$ of the channel, Fig.1, unveils the interplay between surface and bulk properties and allows three main regimes to be identified:

i) the regime $H \ll L \ll \lambda_{a}$, where particles move ballistically in all directions (Fig, 2 a) and the majority of them lay in the proximity of the two walls, eventually sliding 
along them.

ii) in the regime $H \ll \lambda_{a} \ll L$, a diffusive effective motion emerges along the axis channel while the transversal motion is characterized by rebounds between the walls. The snapshot b) of Fig 2 shows that under this condition the accumulation reduces and is no longer dominant.

iii) the regime $\lambda_{a} \ll H$, where the persistence length is smaller than any geometrical scale. As shown in Fig.2p, the phenomenology is similar to the one of a Brownian system at an effective temperature, $T=\gamma U_{0}^{2} / 2 D_{r}$.

As a quantitative measure of accumulation, we plot in Fig 4 the fraction $N_{w} /\left(N-N_{w}\right)$ versus $D_{r}$, by counting the particles contained in the stripes, parallel and adjacent to each boundary, of transversal size $\sigma$. This ratio exhibits a monotonic decreasing behavior with $D_{r}$ towards the Brownian limit, further indicating that the increase of $D_{r}$ depresses the accumulation at the wall.

\section{B. Case $\epsilon>0$}

We now discuss the case where an external force of strength $\epsilon$ pushes the particles towards the right. At variance with the case without drift, $U_{0}$ plays a fundamental role as it combines with the drift $\epsilon / \gamma$. We vary $U_{0}$, keeping $\epsilon=0.5$, and explore the two regimes $\gamma U_{0} \geq \epsilon$ and $\gamma U_{0}<\epsilon$, at different values of $D_{r}$.

In Fig 5 , we show six snapshots of particle configurations at different values of $D_{r}$ and $U_{0}$. Panels a), b) and c) referring to $D_{r}=1.0$ and $U_{0}=1,0.5,0.1$ show a Brownian-like behavior with the effective temperature, $T=\gamma U_{0}^{2} / 2 D_{r}$. In this regime, the Brownian fluctuations are not able to counteract the effect of the bias so that no particle can escape on the left.

As shown in figures $5 \mathrm{a}$ and $5 \mathrm{~b}$ when $\epsilon \lesssim \gamma U_{0}$, the particles may fill vertically the whole sector $x>0$ of the channel, whereas in the opposite regime $\left(\epsilon \gtrsim \gamma U_{0}\right)$, the drift prevails over diffusion creating a sort of "plume" towards the right exit, as illustrated in Fig.5.

The persistent case $D_{r}=0.01$ is shown in panels $\mathrm{d}$ ), e) and f). In panel d), the particles can explore the whole channel despite the bias, on the contrary, when the ratio $\gamma U_{0} / \epsilon$ decreases, the bias prevails, and particles injected at the point $P$ can only explore angles $\beta$ 
such that

$$
|\beta| \leq \tan ^{-1}\left(\frac{\epsilon}{U_{0}}\right)
$$

see panels e) and f). Such a condition is obtained by assuming a less favorable case where the active force has only the $y$-component, thus $\dot{x}=\epsilon, \dot{y}= \pm U_{0}$.

\section{DISTRIBUTION ALONG CHANNEL AXIS}

A successful approximation often employed in the study of the transport of passive particles in narrow channels with non-uniform section is represented by the so called Fick-Jacobs

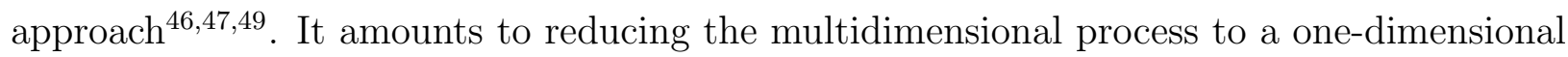
diffusion in the effective potential encoding the channel geometry. Such an approximation is valid whenever the system reaches a steady distribution in the transversal section on a time-scale much shorter than the typical time of the process along the channel axis ${ }^{48 \mid 63 / 64}$. To what extent this homogenization approach is valid for active particles is not clear. Our results show that transversal homogenization is not fulfilled when $D_{r}<\gamma$, because persistent values of the active force favor the accumulation at the walls. In this respect, we numerically study the stationary marginal probability distribution, $p_{\mathrm{st}}(x)$, along the channel axis,

$$
p_{\mathrm{st}}(x)=\frac{1}{w(x)} \int_{0}^{w(x)} d y P_{\mathrm{st}}(x, y)
$$

obtained from the two-dimensional distribution $P_{\mathrm{st}}(x, y)$. Notice that the existence of a stationary state is a consequence of the re-injection that replaces the particles exiting from the boundaries $x= \pm L$. The numerical $p_{\mathrm{st}}(x)$ are reported in Fig. 6 a in the absence of bias and in Fig.6b in the presence of bias, $\epsilon$, for different values of $D_{r}$.

For $\epsilon=0$, panel a), we observe an asymmetry with respect to the center of the channel $(x=0)$ reflecting the narrowing of the section $w(x)$. Indeed, the slant of the upper wall generates an "entropic" drift favoring a larger occupation of the side $x<0$. This entropic effect is more evident for large $D_{r}$ and maximal in the Brownian limit characterized, up to a normalization constant, by a distribution (dashed black line)

$$
p_{s t}(x)=\left\{\begin{array}{lr}
A(a-x) \ln \left(\frac{a+L}{a-x}\right) & x \in[-L, 0] \\
B(a-x) \ln \left(\frac{a-x}{a-L}\right) & x \in[0, L]
\end{array}\right.
$$



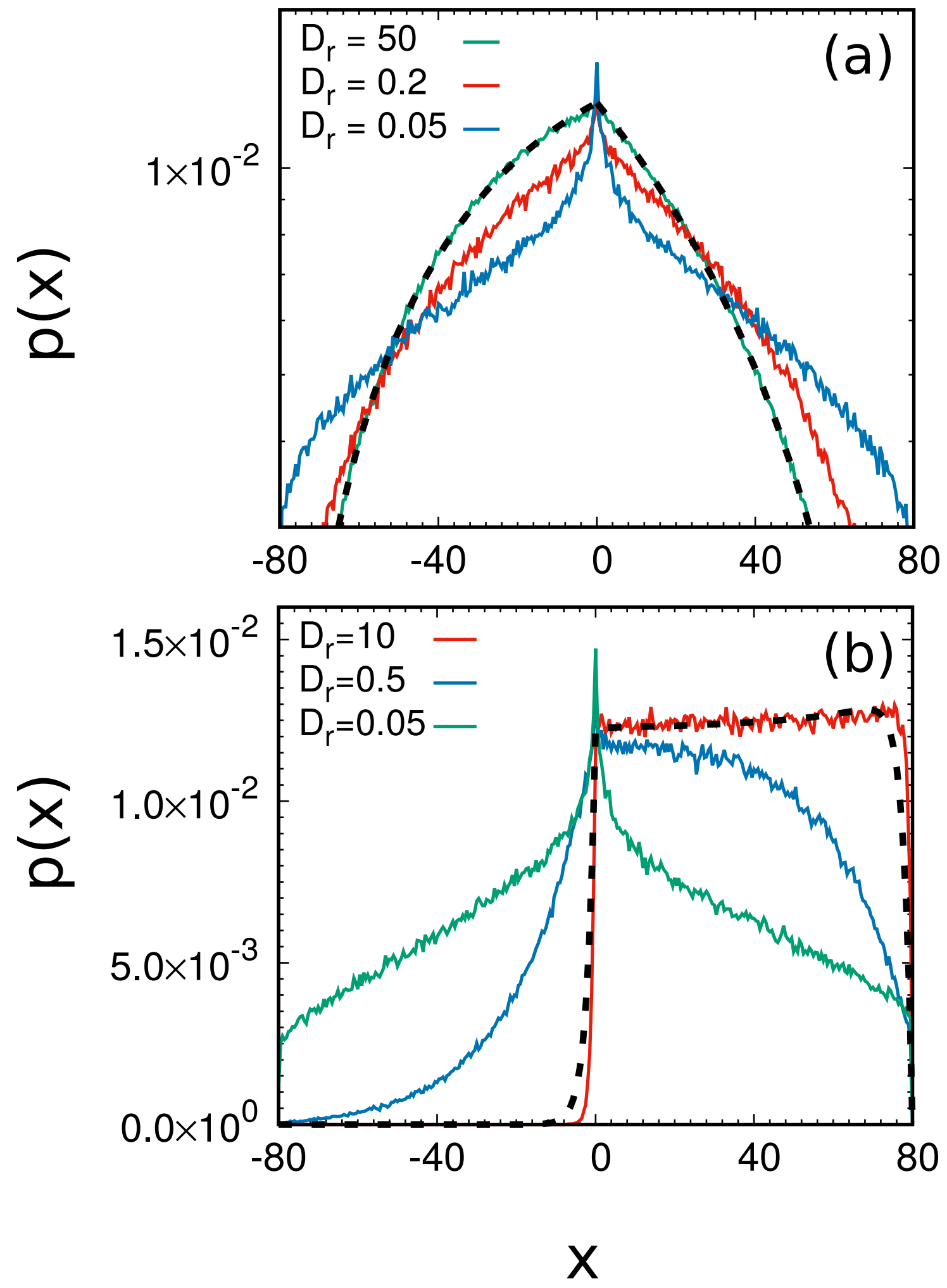

FIG. 6. Stationary marginal space probability distribution, $p_{\mathrm{st}}(x)$, at different values of $D_{r}$. Panel a) refers to $\epsilon=0$, panel b) to $\epsilon>0$; dashed lines represent the Brownian predictions: Eq.(6) for $\epsilon=0$ and Eq.(7) for $\epsilon>0$. System parameters are: $L=80, a=100, H=10, U_{0}=3.0$. 


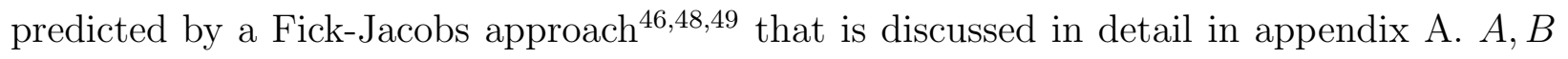
are two coefficients depending on the geometry parameters, $a, L, H$.

It is interesting to remark that formula (6) remains reasonably applicable to active particles till to values around $D_{r}=1$. At smaller $D_{r}$, the entropic drift is contrasted by the persistence of the trajectories and consequently the distribution becomes more symmetric, it also develops a narrow peak near $x=0$, more and more pronounced as $D_{r}$ is reduced. This over-crowding of the region near $x=0$, absent in the Brownian case, is a combined effect of re-injection and persistence that determines the accumulation of the particles pointing towards the walls.

As shown in Fig $6 \mathrm{~b}$, a constant field $\epsilon=0.5$ overwhelms the "entropic" drift and determines a larger density in the region $x>0$. Even in this case, the shape of $p_{\mathrm{st}}(x)$ is strongly influenced by the activity, and again the large $D_{r}$ range recovers the Brownian-like profile

$$
p_{\text {st }}(x) \propto e^{\varepsilon x}(a-x)\left\{\begin{array}{r}
A(\operatorname{Ei}[\varepsilon(a+L)]-\operatorname{Ei}[\varepsilon(a-x)]) \\
x \in[-L, 0] \\
B(\operatorname{Ei}[\varepsilon(a-x)]-\operatorname{Ei}[\varepsilon(a-L)]) \\
x \in[0, L]
\end{array}\right.
$$

which is also derived in Appendix A. In expression (7), we set $\varepsilon=\gamma \epsilon / T$ (with $T=\gamma U_{0}^{2} / 2 D_{r}$ meant as an effective temperature) and $\operatorname{Ei}[\ldots]$ denotes the Exponential Integral function (cfr. pp. 661-662 of Ref. $\frac{65}{}$ ).

We conclude by remarking that in the strong activity regime [blue and green curves in Figs. 6(a) and 6(b)], the active force is able to shadow both the entropic and the bias effects, thus leading to a symmetrization of the profiles.

In Sec IV, we see how the accumulation mechanism of the particles to the walls strongly affects the escape process.

\section{ESCAPE PROCESS OF ACTIVE PARTICLES FROM THE WEDGE}

We study numerically the escape statistics from the wedge, $Q$, for the ensemble of particles initially injected in the neighborhood of $(0, H / 2)$. We define the left and right first passage times, $\tau_{L, R}$, as the first time at which a given particle leaves $Q$ either from the left or from 
the right boundary,

$$
\begin{aligned}
& \tau_{L}=\min _{t}\left\{0<t \leq T_{w} \mid x(t)<-L\right\} \\
& \tau_{R}=\min _{t}\left\{0<t \leq T_{w} \mid x(t)>L\right\} .
\end{aligned}
$$

within a given simulation time window $\left[0, T_{w}\right]$. A convenient choice is $T_{w} \sim 10^{4} / D_{r}$ to allow all the particles to exit in a reasonable simulation time. We investigate the three different dynamical regimes discussed in Sec III and obtain numerically the exit-time distributions, $\Psi_{L, R}(\tau)$, by the histogram method. We start discussing the results in the case of no drift, $\epsilon=0$, and then we consider the driven system, $\epsilon>0$, using the Brownian case as a reference.

\section{A. Active escaping time at $\epsilon=0$}

Figure 7 reports the distributions of first exit times, $\Psi(\tau)$, at different values of $D_{r}$.

When $\lambda_{a} \ll H \ll L$, the majority of the particles spreads in the bulk and their behavior is hardly distinguishable from the one of a swarm of Brownian particles with temperature $\gamma U_{0}^{2} / 2 D_{r}$. Accordingly, the $\Psi(\tau)$ is very similar to the escape time distribution of Brownian particles from the wedge.

When the persistence length is such that, $H \ll \lambda_{a} \ll 2 L$, the situation changes because: i) a large fraction of particles spends much time stuck to the up and bottom boundary, ii) the vertical component of the particle velocity behaves quite "deterministically" producing a bouncing ball effects between the upper and lower boundaries that lasts for a period of the order of the persistence time $e^{60}, t_{a} \sim 1 / D_{r}$. As a result of the "stickiness" of the walls, we observe a sort of dimensional reduction which confers to the $\Psi(\tau)$ a shape strongly deviating from the corresponding Brownian distribution. At first, as seen in both figures $7 \mathrm{a}$ and $7 \mathrm{~b}$, a

pronounced asymmetry of $\Psi(\tau)$ occurs, characterized by the emergence of a fat tail at larger times and a rather steep shoulder at shorter times.

In the regime $H \ll L \ll \lambda_{a}$, particles strongly accumulate along the walls which act as trails guiding the particles to the left or right exit, in a time roughly given by $t_{d} \sim L / U_{0}$. As a consequence, the escape problem reduces to the combination of two one-dimensional escape processes, each occurring along one of the walls. Accordingly, the exit-time distribution becomes extremely peaked near $t_{d}$. The possibility of escaping within $t_{d}$ clearly depends on the initial random orientation of the active force at the injection point. In this respect, we 

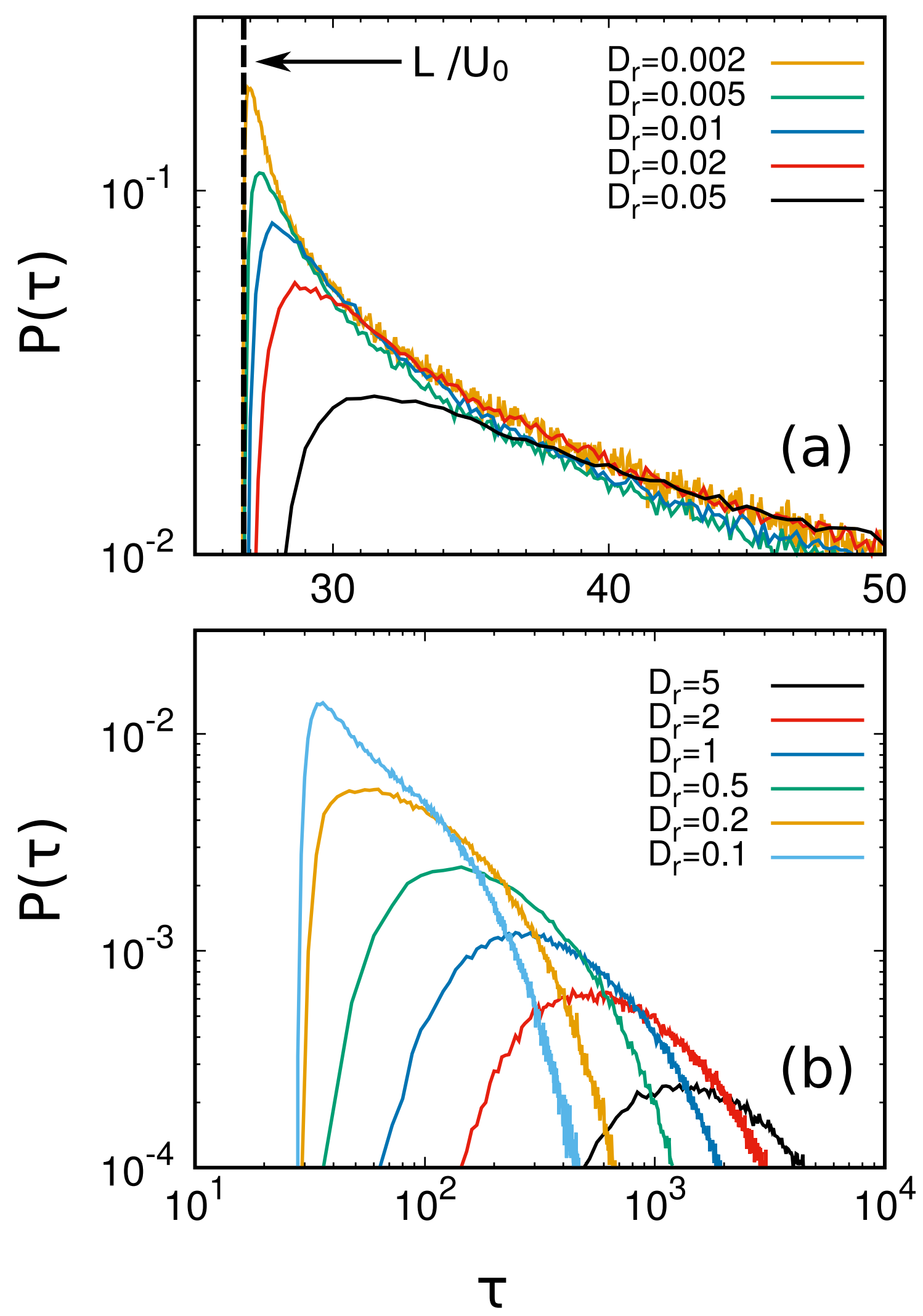

FIG. 7. Escape time distribution, $\Psi(\tau)$, for $\epsilon=0$, computed at selected values of $D_{r}$ in the range $\left[2 \times 10^{3}, 5\right]$, see the legend. Plots have been split in two panels for readability reasons: panel (a) refers to the range $\left[2 \times 10^{-3}, 5 \times 10^{-2}\right]$ while panel (b) to the range $[0.1,5.0]$ Other used parameters are $L=80, a=100, H=10$, and $U_{0}=3$. 


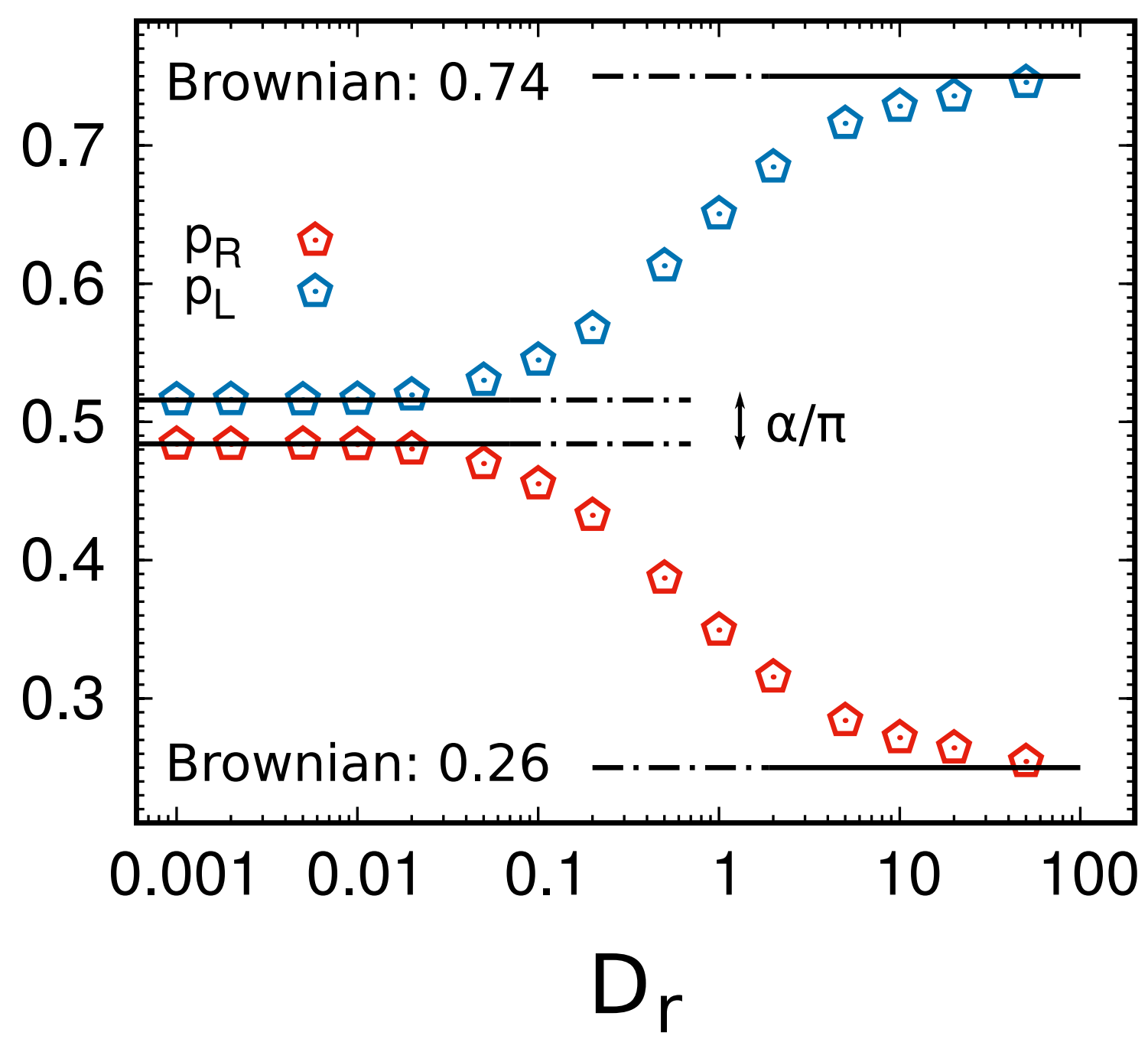

FIG. 8. Dependence of the left/right escape probabilities, $p_{L}$ and $p_{R}$, on $D_{r}$. Black lines indicate the two limiting plateaus: the Brownian prediction from Eq. 10 , and the infinite- $\tau$ prediction given by Eq.(11).

can classify the particles in two groups: group $\mathrm{A}$ is formed by particle with initial direction allowing them to leave the channel in a time, $t \sim t_{d}$, either to the left or to the right, without changing direction. Instead, group B contains particles changing direction at least one time before they reach one of the exits at a larger time. The particles belonging to A, arriving quite at the same time $t_{d}$, contribute to the peak in the $\Psi(\tau)$, while the arrivals of the particles of group B contribute to the long tails. In this regime, the decreasing of $D_{r}$ produces higher and thinner spikes and longer tails.

We plot in Fig.8 the right and left escaping probability, $p_{R}, p_{L}=1-p_{R}$, respectively, obtained by measuring the fraction of exit events from the right and from the left in a long 


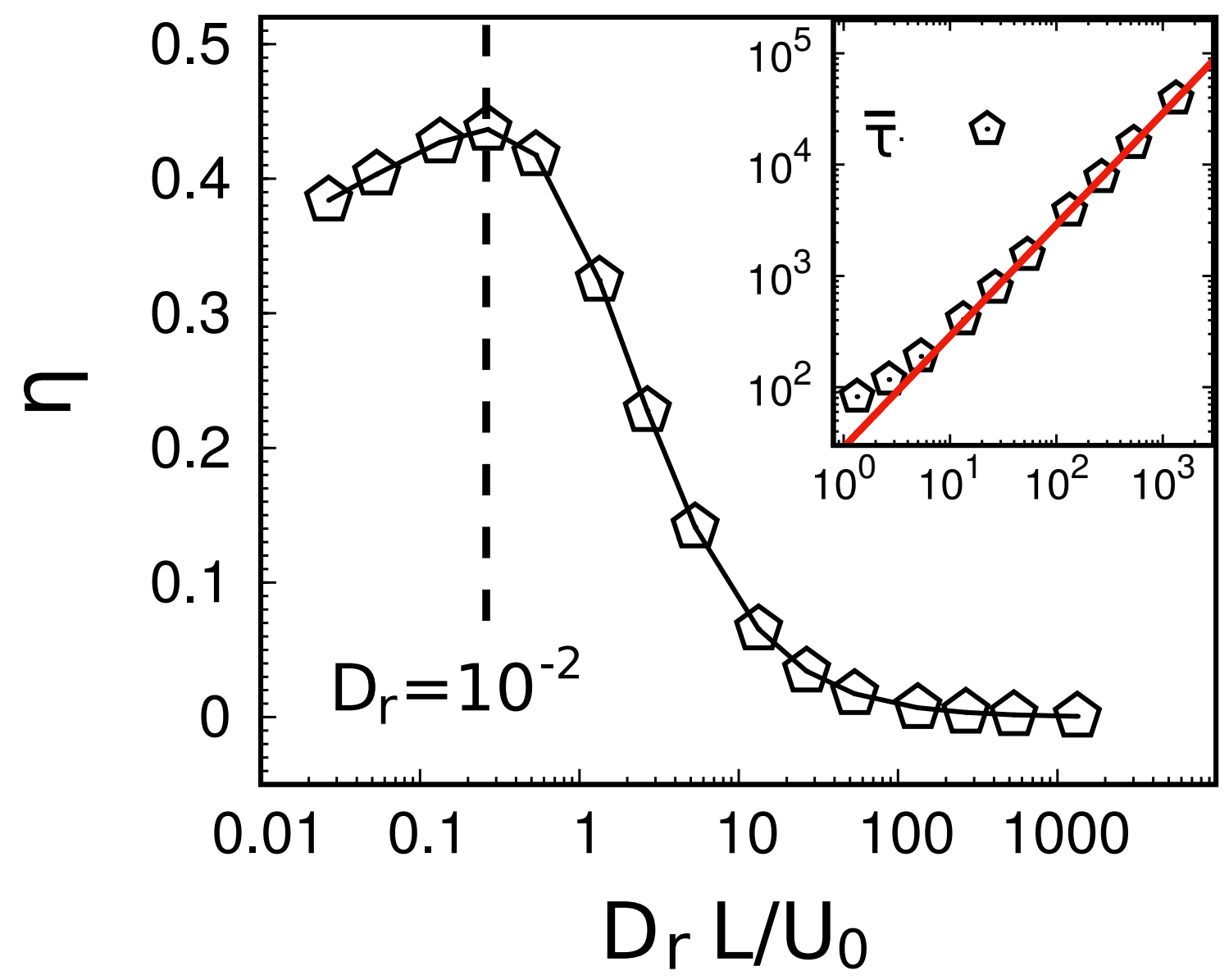

FIG. 9. Efficiency, defined by Eq. (12), as a function of the dimensionless parameter $D_{r} L / U_{0}$, in the case of zero drift $\epsilon=0$. The peak is attained at $D_{r} \approx 10^{-2}$. The inset shows $\bar{\tau}$ (black data) vs. $D_{r} L / U_{0}$ for a comparison with the Brownian result, Eq. 13 . Parameters: $L=80, a=100$, $H=10, U_{0}=3$.

simulation. We see that $p_{R}$ and $p_{L}$ show a clean monotonic behavior as a function of $D_{r}$, converging to two different plateaus for $D_{r} \rightarrow 0$ and $D_{r} \rightarrow \infty$, respectively. The plateau for $D_{r} \gg \gamma$ (Brownian regime) is given by the expression

$$
p_{R}=\frac{\ln (a+L)-\ln a}{\ln (a+L)-\ln (a-L)}=\frac{\ln (1+\mu \tan \alpha)}{\ln \left(\frac{1-\mu \tan \alpha}{1+\mu \tan \alpha}\right)}
$$

where $\mu=L / H$ and $\alpha$ is the wedge angle, see Fig,1. The derivation of the above expression can be found in appendix A, specifically see Eq.A10.

Notice that in the Brownian case, there is not a temperature dependence. For $H=100$ and $L=80$, Eq. 10 provides the values $p_{R} \simeq 0.2675$ which agrees with the simulation value. In this case, $p_{L}$ is much less than $p_{R}$, due to the obvious action of the entropic "drift" 

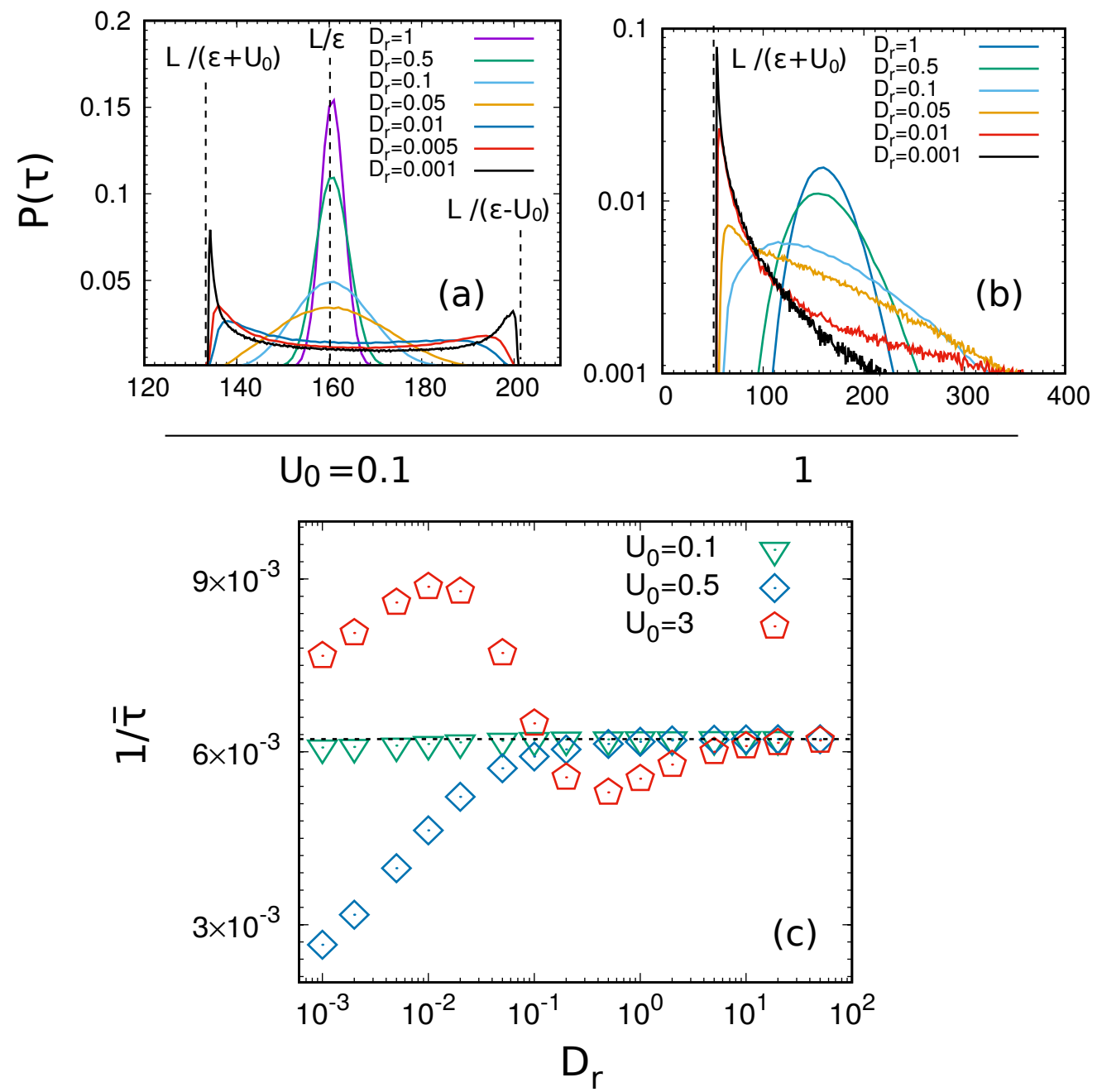

FIG. 10. Panels a) and b): escape time distributions at different values of $D_{r}$ and two different values of $U_{0}=0.1,1$ in the presence of an external bias $\epsilon=0.5$. Panel (c): dependence of $1 / \bar{\tau}$, namely the transport efficiency, on $D_{r}$ at values $U_{0}=0.1,0.5,3$ and $\epsilon=0.5$. Geometry parameters are $L=80, a=100, H=10$.

produced by the wedge geometry which favors the exit to the larger left side. This scenario remains valid up to values of $D_{r} \sim 10$.

If $D_{r}$ further decreases, $p_{L}$ develops a strong dependence on $D_{r}$, indicating that the activity counteracts the entropic drift and facilitates the passage through the narrow side of the channel. In practice, the particle accumulation to the walls has the effect of reducing the entropic barrier. A similar "rectifying" phenomenology has been observed for active Janus particles in periodic channels alternating two wedge compartments . $^{66}$. 
At some value of $D_{r}, p_{R(L)}$ saturates to a value, $p_{R, L}^{*}$

$$
p_{L, R}^{*}=\frac{\pi \pm \alpha}{2 \pi}
$$

Indeed, if the motion of the particles is so persistent to be considered "ballistic", $p_{L}$ and $p_{R}$ strongly depend on the initial re-injection condition. With reference to Fig.1, we can identify two complementary intervals $\mathcal{A}_{R}=[-\pi / 2, \pi / 2-\alpha]$ and $\mathcal{A}_{L}=[\pi / 2-\alpha, 3 \pi / 2]$, for which those active particles emitted with an initial angle either $\theta_{i} \in \mathcal{A}_{R}$ or $\theta_{i} \in \mathcal{A}_{L}$ are bound to exit almost surely either to the left or to the right, respectively. Eq. 111 is the analogue of Eq. 10 in the regime of strong persistence. Of course, always exists a very small fraction of particles whose orientation prevents the exit in a time $t_{a}$, but this fraction is very small and does not affect the exit time statistics except for the short tail.

It is interesting to study the average exit time from the wedge

$$
\bar{\tau}=\int_{0}^{\infty} d \tau \Psi(\tau) \tau
$$

as a function of the control parameters. This observable is able to quantify the transport efficiency and it is relevant to understand if the activity favors or not the emptying of the channel.

We can define the transport efficiency as the ratio

$$
\eta=\frac{L}{U_{0} \bar{\tau}}
$$

between the time, $L / U_{0}$, at which a deterministic motion of velocity $U_{0}$ gains the exit and the mean exit time $\bar{\tau}$.

In Fig 9, we plot $\eta$ for $U_{0}=3$ versus the dimensionless parameter $D_{r} L / U_{0}$. It exhibits a non-monotonous behavior reaching its maximal value at $D_{r} L / U_{0} \approx 0.3$. This reveals the existence of an optimal $D_{r}$ such that the escaping process becomes more efficient, in the specific parameter choice $D_{r} \simeq 0.01$. The increase of the "transport efficiency" can be explained by invoking a sort of "dimensional reduction". Specifically, those particles accumulating at the walls are favored in the exit process because they use the boundaries like trails, thus performing basically a one-dimensional motion along them. This greatly enhances the possibility to find the exit with respect to the case where particles explore the full wedge in order to escape. Ref. ${ }^{44}$ shows that the accumulation near the walls also depends on the hydrodynamics interactions. As a consequence, the efficiency peak in Fig.9 shifts towards larger or smaller values of $D_{r} L / U_{0}$ in the case of pullers or pushers, respectively. 
At very small $D_{r} L / U_{0}$, however $1 / \bar{\tau}$ decreases as a finite fraction of particles, in particular, those hitting normally the walls, almost remain stuck for a time $1 / D_{r}$, (diverging for $D_{r} \rightarrow$ 0 ) thus slowing down their escape process. Again for $D_{r} \gtrsim \gamma$, the system approaches a Brownian regime and $\bar{\tau}$ linearly increases with $D_{r} L / U_{0}$, as shown in the inset of Fig.9. Indeed, the effective temperature $T=\gamma U_{0}^{2} / 2 D_{r}$, which in this case controls the Brownianlike behavior, increases with $D_{r} L / U_{0}$. The inset of Fig, 9 shows also a comparison between the numerical $\bar{\tau}$ and the prediction derived in appendix $\mathrm{A}$ for a Brownian particle at temperature $T=\gamma U_{0}^{2} / 2 D_{r}$

$$
\bar{\tau}=\frac{D_{r} L^{2}}{2 \gamma U_{0}^{2}}\left(1+\frac{a}{2 L} \frac{\ln \left[a^{2} /\left(a^{2}-L^{2}\right)\right]}{\ln [(a+L) /(a-L)]}\right) .
$$

The first term corresponds to the average exit time of a one-dimensional system of length $2 L$, whereas the second one is associated with the entropic barrier and reflects the asymmetry of the channel. As expected, the linear behavior of $\bar{\tau}$ with $D_{r}$ in the effective equilibrium regime is in very good agreement with the prediction.

\section{B. Active escaping time at $\epsilon>0$}

In this section, we analyze how an external driving $\epsilon$ modifies the previous scenario. Consistently with the concept of effective temperature, when $D_{r}$ is large enough we expect a Brownian-like regime and thus an exit time distribution, $\Psi(\tau)$, resembling the corresponding Brownian distribution at temperature $\gamma U_{0}^{2} / 2 D_{r}$, as shown in Fig 10 . In this case $\Psi(\tau)$ is peaked around, $t_{m} \sim \gamma L / \epsilon$, representing the time taken by particles of velocity $\epsilon / \gamma$ to travel a distance $L$. In this regime, the reduction of $U_{0}$ or $D_{r}$ increases the variance of $\Psi(\tau)$. In the Brownian-like regime, the decreasing of $D_{r}$ produces the enhancement of the skewness of $\Psi(\tau)$ and the emergence of long right tails.

A further decrease of $D_{r}$ shifts the peak of $\Psi(\tau)$ to the left and simultaneously the right tail becomes higher, until the mean peak position pins at $t_{m} \sim \gamma L /\left(\gamma U_{0}+\epsilon\right)$. In this regime, the reduction of $D_{r}$ leads only to more pronounced peaks of $\Psi(\tau)$. Indeed, even the particles which move ballistically towards the exit without changing their orientation (group A) cannot reach the exit within the minimal time $\sim \gamma L /\left(\gamma U_{0}+\epsilon\right)$. Depending on the ratio $\gamma U_{0} / \epsilon$ a different phenomenology occurs: i) $\gamma U_{0}<\epsilon$ : a secondary peak of $\Psi(\tau)$ occurs at a value $\gamma L /\left(\epsilon-\gamma U_{0}\right)$ and finally the distribution abruptly drops down. This secondary peak is due to the slow particles whose orientation is opposed to the $x$-direction where the constant 
force is directed. ii) $\gamma U_{0}>\epsilon$ : the first peak simply becomes higher but the second vanishes.

The efficiency $\eta$ of the transport for $\epsilon>0$ is defined by replacing $U_{0} \rightarrow U_{0}+\epsilon$ in Eq.(12), but as a matter of fact, the inverse of the mean exit time is already an estimate of $\eta$. For this reason Fig, 10 e reports directly $1 / \bar{\tau}$ versus $D_{r}$ to quantify the channel emptying at three values of $U_{0}$. In the case $\epsilon>\gamma U_{0}$, the increasing of $D_{r}$, i.e. the decreasing of the effective temperature, leads to a monotonic growth of $1 / \bar{\tau}$, until saturation is reached and the system behaves as a Brownian one. In this regime, the activity reduces the efficiency of the transport process. Indeed, despite some particles travel towards the exit with more facility, also several particles move in the opposite direction, employing a long time before leaving the wedge. Clearly, the reduction effect becomes relevant only when $U_{0}$ is comparable with $\epsilon$ (blue diamond data) otherwise (when $\gamma U_{0} \ll \epsilon$ ) the particles leave the wedge only because of the driving $\epsilon$ (green triangle data).

The situation is more interesting in the opposite regime, where $\epsilon<\gamma U_{0}$. In this case, $1 / \bar{\tau}$, reveals a non-monotonic behavior in terms of $D_{r}$. Starting from the Brownian saturation value, a first decreasing of $D_{r}$ produces a reduction of $1 / \bar{\tau}$, until a minimum value; a situation resembling the previous one for $\epsilon>\gamma U_{0}$. Nevertheless, a further decrease of $D_{r}$, produces the increasing of $1 / \bar{\tau}$, up to a maximum value which reveals an increase of the transport efficiency for some value of $D_{r}$. This persistence maximizes the efficiency of the transport process, since for smaller $D_{r}$ a decrease of $1 / \bar{\tau}$ occurs, due to the same mechanism already discussed in the case $\epsilon=0$.

As a consequence, the activity can be seen as an optimization mechanism, which reduces the time employed by microswimmers to reach the exit of the channel, also in the presence of a constant driving force. A condition for this scenario implies that the activity strength has to be stronger than the amplitude of the external driving.

\section{CONCLUSIONS}

In this work, we studied the escape process of a system of ABP particles from an openwedge channel in the absence and in the presence of an external driving force. The comparison with the Brownian system counterpart shows that activity facilitates the escaping from the narrow exit and competes against the entropic force. We also found the existence of an optimum value of the persistence time which maximizes the channel emptying. The physical 
mechanism which improves the efficiency is the effective attraction exerted by the bottom and upper walls on the active particles which leads to a depletion of the inner region. The majority of the particles accumulate in a narrow region near each wall and as a consequence, the section-dependent entropic force is strongly reduced. The resulting motion of the particles is effectively one-dimensional and controlled by the competition between the active force and the external field. Somehow, the activity is able to operate a sort of dimensional reduction, since makes surface effects prevailing over the bulk properties.

The present treatment shows that the extension of the Fick-Jacobs approximation to the active case is possible only for small values of the persistent time and activity strength ${ }^{50}$. When it happens, the far-equilibrium feature of active particles do not emerge and the Brownian theory with an effective temperature agrees with numerical data. On the contrary, when the active force is very persistent and large, our study of particle distributions shows that the main hypothesis underlying the Fick-Jacobs approximation breaks down, since the density is not homogeneous in each channel section, as an effect of the accumulation near the walls. Quantifying how the effective entropic barrier is reduced by this mechanism could represent an intriguing next point in understanding biological transport processes.

This work suggests that the active transport can be facilitated or even optimized by a proper design of the channel surface. This can be interpreted as further manifestation of "ratcheting mechanism" that can be observed as soon as active particles experience confining forces breaking the left-right symmetry ${ }^{66}$.

We point out that the presence of inter-particle interactions could drastically affect the transport properties in the channel. Indeed, as mentioned in the introduction, interactions could promote clustering until MIPS takes place in the regime of strong active forces. Assessing the effects of MIPS on the mean exit time from the channel will certainly constitute an interesting subject for future investigations.

\section{Appendix A: Brownian case}

In this appendix, we describe the spreading of Brownian particles distribution, $P(x, t)$, along the axis of the channel $Q$ in terms of Fick-Jacobs approach 46 . Fick-Jacobs theory is applicable to channels with variable sections, $w(x)$, provided the particle distribution attains its steady form along the $y$-direction on a time-scale much shorter than the time 
scale associated with the longitudinal motion (transversal homogenization). However, this condition, that can be fulfilled by Brownian particles, is violated by the active particles in those regimes where accumulation to the boundaries is not negligible.

In the Brownian regimes, the strong confinement in the lateral direction allows the diffusion of the particles along the channel to be described in a quasi-one-dimensional equation

$$
\begin{aligned}
& \frac{\partial P}{\partial t}+\frac{\partial J}{\partial x}=k \delta(x) \\
& P(L, t)=P(-L, t)=0
\end{aligned}
$$

where the last two equations mathematically implement the absorbing boundary conditions at $x= \pm L$. The Dirac-delta source with amplitude $k$ accounts for the instantaneous reinjection, at $x=0$, of those particles leaving the channel from the boundaries $x= \pm L$.

The current

$$
J(x, t)=-\frac{T}{\gamma} e^{\gamma \epsilon x / T} w(x) \frac{\partial}{\partial x}\left[e^{-\gamma \epsilon x / T} \frac{P(x, t)}{w(x)}\right]
$$

describes to a good approximation the longitudinal transport along a channel of variable section $w(x)=H-H x / a$, in the presence of a constant field (bias) of strength $\epsilon$ acting along the channel axis. In the following, we set

$$
D_{0}=\frac{T}{\gamma} \quad, \quad \varepsilon=\frac{\gamma \epsilon}{T} .
$$

The balance between absorption and re-injection preserves the number of particles and gives rise to a steady-state characterized by the equality

$$
J(L)-J(-L)=k,
$$

stating that the re-injection rate balances the loss fluxes at the boundaries.

The stationary distribution satisfies the equation

$$
-D_{0} \frac{\partial}{\partial x}\left[w(x) e^{\varepsilon x} \frac{\partial}{\partial x} e^{-\varepsilon x} \frac{P}{w(x)}\right]=k \delta(x) .
$$

The presence of the Dirac $\delta(x)$ requires the splitting of the solution over the two domains $[-L, 0]$ and $[0, L]$, such that:

$$
P(x)=\left\{\begin{array}{lr}
A Y_{1}(x) & -L \leq x<0 \\
B Y_{2}(x) & 0 \leq x \leq L
\end{array}\right.
$$


where

$$
Y_{1}(x)=e^{\varepsilon x} w(x) \int_{-L}^{x} d u \frac{e^{-\varepsilon u}}{w(u)}
$$

and

$$
Y_{2}(x)=e^{\varepsilon x} w(x) \int_{x}^{L} d u \frac{e^{-\varepsilon u}}{w(u)}
$$

are the fundamental solutions of the homogeneous equation $(k=0)$ satisfying the boundary conditions $Y_{1}(-L)=Y_{2}(L)=0$. The coefficients $A, B$ are determined by imposing the continuity condition, $P\left(0^{+}\right)=P\left(0^{-}\right)$, and from integrating both members of Eq. A4 over the interval $[-\Delta, \Delta]$, then taking $\Delta \rightarrow 0$.

The solution of these constraints provides,

$$
A=\int_{0}^{L} d x \frac{e^{-\varepsilon x}}{w(x)} \quad, \quad B=\int_{-L}^{0} d x \frac{e^{-\varepsilon x}}{w(x)} .
$$

A little algebraic manipulation yields the explicit result

$$
\begin{aligned}
& A=\operatorname{Ei}[\varepsilon a]-\operatorname{Ei}[\varepsilon(a-L)] \\
& B=\operatorname{Ei}[\varepsilon(a+L)]-\operatorname{Ei}[\varepsilon a],
\end{aligned}
$$

where $\operatorname{Ei}[u]$ is the Exponential Integral of argument $u^{65}$. The final expression reads

$$
P(x) \propto e^{\varepsilon x}(a-x)\left\{\begin{array}{c}
A(\operatorname{Ei}[\varepsilon(a+L)]-\operatorname{Ei}[\varepsilon(a-x)]) \\
\text { when } x \in[-L, 0] \\
B(\operatorname{Ei}[\varepsilon(a-x)]-\operatorname{Ei}[\varepsilon(a-L)]) \\
\text { when } x \in[0, L]
\end{array}\right.
$$

up to a normalization constant such that the integral of $P(x)$ over $[-L, L]$ is set to 1 .

These expressions drastically simplify in the zero-field limit, $\epsilon=0$,

$$
P(x)=C(a-x) \begin{cases}A \ln \left[\frac{a+L}{a-x}\right] & x \in[-L, 0] \\ B \ln \left[\frac{a-x}{a-L}\right] & x \in[0, L]\end{cases}
$$

with $A=\ln [(a+L) / a], B=\ln [a /(a-L)]$ and a normalization constant

$$
C^{-1}=\frac{L}{4}\left[2 a \ln \left(\frac{a^{2}}{a^{2}-L^{2}}\right)+L \ln \left(\frac{a+L}{a-L}\right)\right] .
$$

Now, we are in the position to derive formula 10 for the ratio $p_{R}$, which determines the Brownian limit in Fig, 8 . The value of $p_{R}$ can be estimated in term of the fluxes at the 
boundaries, which, due to the fact that $P( \pm L)=0$ are only proportional to the derivatives of $P$ at $x= \pm L$, i.e. $J( \pm L)=-D_{0} P^{\prime}( \pm L)$. Then $p_{R}$ is obtained as the fraction of the flux to the right over the total flux

$$
p_{R}=\frac{J(L)}{J(L)+J(-L)}=\frac{\ln (a+L)-\ln (a)}{\ln (a+L)-\ln (a-L)} .
$$

The average first-arrival time at the boundaries $\pm L$, for a particle released at $x$, is related to the survival probability, $S(x, t)$, by the integral 67

$$
\bar{\tau}(x)=\int_{0}^{\infty} d t S(x, t) .
$$

By definition $S(x, t)$ is the probability that the particle has not yet left the interval $[-L, L]$ at the time $t$ and it is known to satisfy the backward Fokker-Planck equation 67

$$
\frac{\partial S}{\partial t}=\frac{D_{0}}{w(x)} \frac{\partial}{\partial x}\left[w(x) \frac{\partial S}{\partial x}\right]
$$

with the boundary conditions $S( \pm L, t)=0$. To obtain a differential equation for $\bar{\tau}(x)$ it is sufficient to integrate Eq.A11) in the interval $0 \leq t<\infty$, and taking into account that $S(x, \infty)=0$ and $S(x, 0)=1$, thus

$$
\frac{d}{d x}\left[w(x) \frac{d \bar{\tau}}{d x}\right]=-\frac{w(x)}{D_{0}},
$$

that has to be solved with the obvious boundary values $\bar{\tau}( \pm L)=0$, stating that particle emitted at the boundary are instantaneously absorbed. We are interested in the the average arrival time from $x=0$, then the searched solution is

$$
\bar{\tau}(x=0)=\frac{L^{2}}{4 D_{0}}+\frac{a L}{2 D_{0}} \frac{\ln \left[a^{2} /\left(a^{2}-L^{2}\right)\right]}{\ln [(a+L) /(a-L)]}
$$

which is exactly Eq. 13 .

\section{REFERENCES}

${ }^{1}$ H. Berg, E. Coli in Motion, Springer Science \& Business Media, 2008.

${ }^{2}$ J. R. Blake and M. A. Sleigh, Biol. Rev., 1974, 49, 85-125.

${ }^{3}$ D. Woolley, Reproduction, 2003, 126, 259-270. 
${ }^{4}$ M. Poujade, E. Grasland-Mongrain, A. Hertzog, J. Jouanneau, P. Chavrier, B. Ladoux, A. Buguin and P. Silberzan, Proc. Natl. Acad. Sci. USA, 2007, 104, 15988-15993.

${ }^{5}$ S. Köhler, V. Schaller and A. R. Bausch, Nature materials, 2011, 10, 462.

${ }^{6}$ A. Walther and A. H. Muller, Chem. Rev., 2013, 113, 5194-5261.

${ }^{7}$ M. Lattuada and T. Hatton, Nano Today, 2011, 6, 286-308.

${ }^{8}$ C. Bechinger, R. Di Leonardo, H. Lowen, C. Reichhardt and G. Volpe, Rev. Mod. Phys., 2016, 045006(50).

${ }^{9}$ P. Romanczuk, M. Bär, W. Ebeling, B. Lindner and L. Schimansky-Geier, Eur. Phys. J. Special Topics, 2012, 202, 1-162.

${ }^{10}$ M. Marchetti, J. Joanny, S. Ramaswamy, T. Liverpool, J. Prost, M. Rao and R. A. Simha, Rev. Mod. Phys., 2013, 85, 1143-1189.

${ }^{11}$ S. Ramaswamy, Annu. Rev. Condens. Matter Phys., 2010, 1, 323-345.

${ }^{12}$ R. Nash, R. Adhikari, J. Tailleur and M. Cates, Phys. Rev. Lett., 2010, 104, 258101.

${ }^{13}$ J. Tailleur and M. Cates, Phys. Rev. Lett., 2008, 100, 218103.

${ }^{14}$ F. J. Sevilla and L. A. G. Nava, Phys. Rev. E, 2014, 90, 022130.

${ }^{15}$ B. ten Hagen, S. van Teeffelen and H. Löwen, J. Phys. Condens. Matter, 2011, 23, 194119.

${ }^{16}$ G. Szamel, Phys. Rev. E, 2014, 90, 012111.

${ }^{17}$ S. Das, G. Gompper and R. Winkler, New J. Phys., 2018, 20, 015001.

${ }^{18}$ U. Marconi Marini Bettolo and C. Maggi, Soft Matter, 2015, 11, 8768-8781.

${ }^{19}$ G. Miño, M. Baabour, R. Chertcoff, G. Gutkind, E. Clément, H. Auradou and I. Ippolito, Adv. Microbiol., 2018, 8, 451-464.

${ }^{20}$ L. Caprini and U. Marconi Marini Bettolo, Soft Matter, 2018, 14, 9044-9054.

${ }^{21}$ H. Wensink and H. Löwen, Phys. Rev. E, 2008, 78, 031409.

${ }^{22}$ A. Kaiser, H. Wensink and H. Löwen, Phys. Rev. Lett., 2012, 108, 268307.

${ }^{23}$ Y. Fily, A. Baskaran and M. Hagan, Soft Matter, 2014, 10, 5609-5617.

${ }^{24}$ J. Elgeti and G. Gompper, Europhys. Lett., 2015, 109, 58003.

${ }^{25}$ E. J. and G. G., Europhys. Lett., 2013, 101, 48003.

${ }^{26}$ E. Fodor, C. Nardini, M. Cates, J. Tailleur, P. Visco and F. van Wijland, Phys. Rev. Lett., 2016, 117, 038103.

${ }^{27}$ U. Marconi Marini Bettolo, A. Puglisi and C. Maggi, Sci. Rep., 2017, 7, 46496.

${ }^{28}$ L. Caprini, U. Marconi Marini Bettolo and A. Vulpiani, J. Stat. Mech.: Theory and Exp., 2018, 2018, 033203. 
${ }^{29}$ S. C. Takatori, R. De Dier, J. Vermant and J. F. Brady, Nature Commun., 2016, 7, 10694.

${ }^{30}$ L. Caprini, U. Marconi Marini Bettolo and A. Puglisi, Sci. Rep., 2019, 9, 1386.

${ }^{31}$ L. Caprini, U. Marini Bettolo Marconi, A. Puglisi and A. Vulpiani, J. Chem. Phys., 2019, 150, 024902 .

${ }^{32}$ Y. Fily and M. Marchetti, Phys. Rev. Lett., 2012, 108, 235702.

${ }^{33}$ I. Buttinoni, J. Bialké, F. Kümmel, H. Löwen, C. Bechinger and T. Speck, Phys. Rev. Lett., 2013, 110, 238301.

${ }^{34}$ J. Bialké, T. Speck and H. Löwen, J. Non-Cryst. Solids, 2015, 407, 367-375.

${ }^{35}$ M. E. Cates and J. Tailleur, Annu. Rev. Condens. Matter Phys., 2015, 6, 219-244.

${ }^{36}$ T. Speck, The European Physical Journal Special Topics, 2016, 225, 2287-2299.

${ }^{37}$ E. Tjhung, C. Nardini and M. Cates, Phys. Rev. X, 2018, 8, 031080.

${ }^{38}$ P. Digregorio, D. Levis, A. Suma, L. F. Cugliandolo, G. Gonnella and I. Pagonabarraga, Phys. Rev. Lett., 2018, 121, 098003.

${ }^{39}$ D. Ribet and P. Cossart, Microbes and Infection, 2015, 17, 173-183.

${ }^{40}$ K. Malakar, V. Jemseena, A. Kundu, K. Kumar, S. Sabhapandit, S. Majumdar, S. Redner and A. Dhar, J. Stat. Mech. Theory Exp., 2018, 2018, 043215.

${ }^{41}$ G. H. Weiss, Physica A, 2002, 311, 381-410.

${ }^{42}$ L. Angelani, R. Di Leonardo and M. Paoluzzi, Eur. Phys. J. E, 2014, 37, 59.

${ }^{43}$ A. Scacchi and A. Sharma, Mol. Phys., 2018, 116, 460-464.

${ }^{44}$ P. Malgaretti and H. Stark, J. Chem. Phys., 2017, 146, 174901.

${ }^{45}$ J. chun Wu, Q. Chen and B. quan Ai, Phys. Lett. A, 2015, 379, 3025 - 3028.

${ }^{46}$ M. H. Jacobs, Diffusion processes, Springer, 1935, pp. 1-145.

${ }^{47}$ R. Zwanzig, J. Phys. Chem., 1992, 96, 3926-3930.

${ }^{48}$ D. Reguera and J. M. Rubí, Phys. Rev. E, 2001, 64, 061106.

${ }^{49}$ P. Burada, P. Hnggi, F. Marchesoni, G. Schmid and P. Talkner, ChemPhysChem, 10, $45-54$.

${ }^{50}$ M. Sandoval and L. Dagdug, Phys. Rev. E, 2014, 90, 062711.

${ }^{51}$ F. J. Sevilla, A. V. Arzola and E. P. Cital, Phys. Rev. E, 2019, 99, 012145.

${ }^{52}$ Y. Fily, arXiv preprint arXiv:1812.05698, 2018.

${ }^{53}$ A. Sharma, R. Wittmann and J. Brader, Phys. Rev. E, 2017, 95, 012115.

${ }^{54}$ C. Bechinger, F. Sciortino and P. Ziherl, Physics of complex colloids, IOS Press, 2013, vol. 184 . 
${ }^{55}$ P. Romanczuk and L. Schimansky-Geier, Phys. Rev. Lett., 2011, 106, 230601.

${ }^{56} \mathrm{R}$. Toral and P. Colet, Stochastic numerical methods: an introduction for students and scientists, John Wiley \& Sons, 2014.

${ }^{57}$ C. Maggi, U. M. B. Marconi, N. Gnan and R. Di Leonardo, Sci. Rep., 2015, 5, 10742.

${ }^{58}$ C. G. Wagner, M. F. Hagan and A. Baskaran, J. Stat. Mech.: Theory and Exp., 2017, 2017, 043203.

${ }^{59}$ L. Angelani, J. Phys. A: Math. and Theor., 2017, 50, 325601.

${ }^{60}$ C. Lee, New J Phys., 2013, 15, 055007.

${ }^{61}$ R. G. Winkler, A. Wysocki and G. Gompper, Soft Matter, 2015, 11, 6680-6691.

${ }^{62}$ C. Kurzthaler, S. Leitmann and T. Franosch, Sci. Rep., 2016, 6, 36702.

${ }^{63}$ P. S. Burada, G. Schmid, D. Reguera, J. M. Rubí and P. Hänggi, Phys. Rev. E, 2007, 75, $051111(8)$.

${ }^{64}$ G. Forte, F. Cecconi and A. Vulpiani, Phys. Rev. E, 2014, 90, 062110(10).

${ }^{65}$ G. Arfken and H. Weber, Mathematical methods for physicists, 2001.

${ }^{66}$ P. K. Ghosh, V. R. Misko, F. Marchesoni and F. Nori, Phys. Rev. Lett., 2013, 110, 268301(5).

${ }^{67}$ C. Gardiner, Handbook of Stochastic: For the Natural and Social Sciences, Springer, Berlin, 2009, vol. 4. 